\title{
Cannulated Screw and Cable are Superior to Modified Tension Band in the Treatment of Transverse Patella Fractures
}

\author{
Yun Tian MD, Fang Zhou MD, Hongquan Ji MD, \\ Zhishan Zhang MD, Yan Guo MD
}

Received: 8 November 2010/Accepted: 29 April 2011/Published online: 15 May 2011

(c) The Author(s) 2011. This article is published with open access at Springerlink.com

\begin{abstract}
Background Although the modified tension band technique (eg, tension band supplemented by longitudinal Kirschner wires) has long been the mainstay for fixation of transverse fractures of the patella, it has shortcomings, such as bad reduction, loosening of implants, and skin irritation. Questions/purposes We conducted a retrospective comparison of the modified tension band technique and the titanium cable-cannulated screw tension band technique.

Patients and Methods We retrospectively reviewed 101 patients aged 22 to 85 years (mean, 56.6 years) with AO/OTA 34-C1 fractures $(n=68)$ and 34-C2 fractures $(n=33)$. Fifty-two patients were in the modified tension band group and 49 were in the titanium cable-cannulated screw tension band group. Followup was at least 1 year (range, 1-3 years). Comparison criteria were fracture reduction, fracture healing time, and the Iowa score for knee function.

Results The titanium cable-cannulated screw tension band group showed improved fracture reduction, reduced healing time, and better Iowa score, compared with the modified tension band group. In the modified tension band group, eight patients experienced wire migration, three of

\footnotetext{
Each author certifies that he or she has no commercial associations (eg, consultancies, stock ownership, equity interest, patent/licensing arrangements, etc) that might pose a conflict of interest in connection with the submitted article.

Each author certifies that his or her institution approved the human protocol for this investigation, that all investigations were conducted in conformity with ethical principles of research, and that informed consent for participation in the study was obtained.
}

Y. Tian ( () , F. Zhou, H. Ji, Z. Zhang, Y. Guo

Department of Orthopaedics, Third Hospital of Peking

University, Beijing 100191, China

e-mail: tianyunbj@sina.com these requiring a second operation. There were no complications in the titanium cable-cannulated screw tension band group.

Conclusions The titanium cable-cannulated screw tension band technique showed superior results and should be considered as an alternative method for treatment of transverse patellar fractures.

Level of Evidence Level III, therapeutic study. See the Guidelines for Authors for a complete description of levels of evidence.

\section{Introduction}

Historically, the treatment of patellar fractures has undergone many changes in operative methods $[1,4,5,7]$. In the 1950s, the principle of using a modified tension band technique for treatment of patellar fractures was first proposed and then subsequently recommended as the primary treatment method for patellar fractures [5, 6, 15]. The principle of the tension band technique is to convert the tension forces acting on the anterior surface into compression forces at the articular surface. This technique can substantially improve results because of its reliable fixation and allowance of early joint motion. However, this technique still has some shortcomings. First, the tension band is supplemented by longitudinal smooth Kirschner wires (K-wires) that bear the risk of loosening and migration [8, 15]. Second, direct interfracture compression cannot be achieved with the $\mathrm{K}$-wire, and additionally, there is the potential of skin irritation $[3,5,13]$. We have adopted what we perceive to be an improved technique, the titanium cable-cannulated screw tension band construct, to remedy the shortcomings of the modified $\mathrm{K}$-wire tension band in treating patellar fractures. 
We compared the titanium cable-cannulated screw tension band technique with the modified tension band technique to see whether the new technique could (1) achieve better reduction and direct interfragmentary compression force; (2) shorten fracture healing time; (3) decrease complications, such as loosening of implants and skin irritations; and (4) achieve better knee function.

\section{Patients and Methods}

This retrospective study included 101 patients (48 men, 53 women) aged 22 to 85 years (mean, 56.6 years) operated on between January 2006 and June 2009. From January 2006 to December 2006, all patients were treated with the modified tension band method. From January 2007 to June 2009, we introduced the titanium cable-cannulated tension band technique. Patient selection was influenced by cost as the titanium cable-cannulated technique was offered only to patients who agreed to pay for this higher-cost procedure. All injuries were enclosed and included slip and fall accidents (94 patients) and car accidents (seven patients). Operations were considered when the articular displacement was greater than $2 \mathrm{~mm}$ or fragment separation was greater than $3 \mathrm{~mm}$ on radiography. The time from fracture to the operation was 1 to 7 days (mean, 3 days). The inclusion criteria were (1) AO/OTA [10] 34-C1 fractures, ie, patellar fractures primarily with a transverse fracture line; and (2) AO/OTA 34-C2 fractures, ie, transverse fractures with a single additional fragment created by a longitudinal fracture line. The exclusion criteria were AO/OTA 34-C3 fractures, ie, comminuted fractures. Twelve patients were lost to followup. Patients were divided into either a titanium cable-cannulated screw tension band group or a modified K-wire tension band group, according to their operation. There were 49 patients in the titanium cable-cannulated screw tension band group (19 men, 30 women) with a mean age of 57.12 years (range, 22-83 years), and there were 52 patients in the K-wire tension band group (29 men, 23 women) with a mean age of 56.12 years (range, 26-82 years). Univariate $t$ tests indicated there were no differences in age, gender, mechanism of injury, fracture classification, and interfragmentary gap between the two groups (Table 1). Followup was at least 1 year (range, 1-3 years).

The operations were performed via an anterior longitudinal incision. After sectioning the skin and subcutaneous tissue followed by fully exposing the patella and its sides, we confirmed the quadriceps femoris aponeurosis and the ligament patellae were adherent to the patellar surface. We examined the fracture and condition of the bilateral aponeuroses and joint capsule, removed fracture chips or any intraarticular hematoma, and rinsed the articular cavity. In the AO/OTA 34C2 fractures, the longitudinal fracture was fixed by one lag screw inserted perpendicularly to the fracture line. With the knee in extension, we clamped the fragments to reduce the fracture with a large towel clamp. After we observed the reduction of articular surface under intraoperative fluoroscopy, we drilled two K-wires (2.0-mm diameter) in parallel from the lower pole to the superior pole of the patella, with a $2-\mathrm{cm}$ space and a 5- to $10-\mathrm{mm}$ distance from the patellar articular surface. The $\mathrm{K}$-wires penetrated the cortex of the superior patellar pole. The position of the K-wire was determined using intraoperative fluoroscopy.

In the modified tension band group, we bent the upper end of the K-wire into hooks and threaded a 1.2-mm steel wire through the upper hooks and lower end of the K-wire to form a figure-of-eight tension band. We then tightened the steel wire using a wire cutter, hid the upper hooks of the

Table 1. Differences between the two groups

\begin{tabular}{lllc}
\hline Variable & $\begin{array}{l}\text { Modified Kirschner } \\
\text { wire tension band }\end{array}$ & $\begin{array}{l}\text { Titanium cable-cannulated } \\
\text { screw tension band }\end{array}$ & Chi square/t value \\
\hline $\begin{array}{l}\text { Age (years)* } \\
\text { Gender }\end{array}$ & $56.12 \pm 16.64$ & $57.12 \pm 15.00$ & -0.319 \\
$\quad$ Male & 29 & 19 & 2.92 \\
$\quad$ Female & 23 & 30 & 0.75 \\
Injury reason & 48 & 46 & 0.285 \\
$\quad$ Slip and fall & 4 & 3 & 0.593 \\
Car accident & & & 2.869 \\
AO/OTA classification & 39 & 29 & -0.684 \\
$\quad 34-C 1$ & 13 & 20 & 0.09 \\
34-C2 & $15.85 \pm 7.95$ & $16.78 \pm 5.37$ & 0.495 \\
Interfragmentary gap (mm)* & & 46 & \\
\hline
\end{tabular}

* Values are expressed as mean $\pm \mathrm{SD}$; the remaining values are expressed as number of patients. 

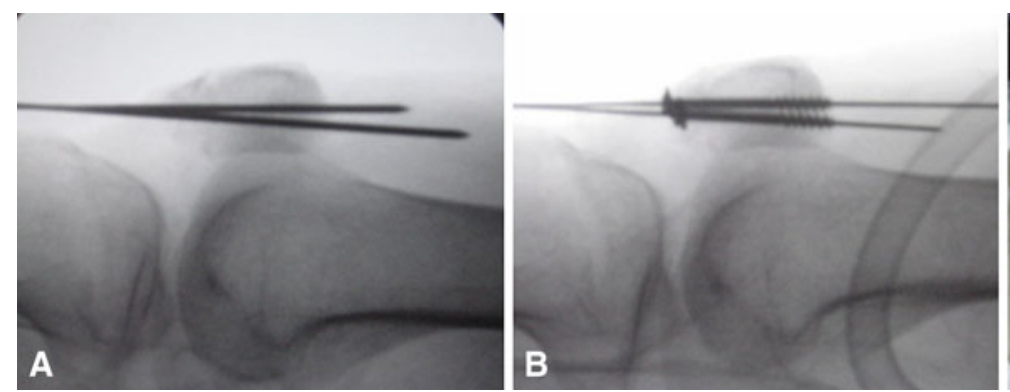

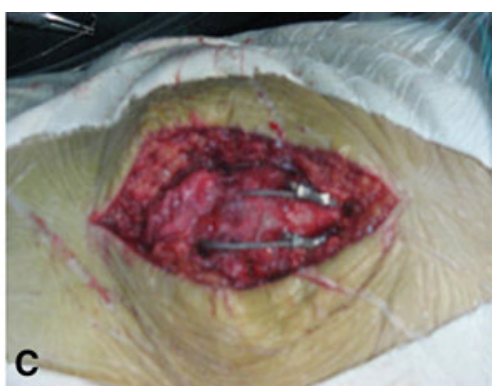

reducing the fracture. (B) Two 4.0-mm-diameter cannulated screws are screwed along the guide wire. $(\mathbf{C})$ Titanium cables are threaded through the cannulated screws and tightened anterior to the patella.
$\mathrm{K}$-wires in the patella, and reobserved reduction of the articular surface by intraoperative fluoroscopy. If the reduction was satisfactory, we cut off the tails of the $\mathrm{K}$-wires and the steel wire.

In the titanium cable-cannulated screw tension band group, one K-wire was first removed and a cannulated screw guide pin was inserted along the pin tract. We drilled along the guide wire with a cannulated bit and, after measuring its depth, screwed a 4.0-mm titanium cannulated compression screw (Synthes Inc, West Chester, PA, USA) along the guide wire. The screw head remained proud of the patella cortex, and the proximal end of the screw should be close to, or embedded within, the patella. We then screwed in the second cannulated screw and alternately tightened the screws. We removed the guide wire and threaded a 1.3-mm-diameter titanium cable (consisting of a total of nine strands; Zimmer Inc, Warsaw, IN, USA) through each of the cannulated screws. We then tightened the titanium cable anterior to the patella to form the tension band, fixed the titanium cable with cable clamps, and cut off the excessive titanium cable (Fig. 1).

Patients performed quadriceps femoris contraction exercises soon after the operation, passive joint flexion and extension exercises 2 days after the operation, and active joint flexion and extension exercises 7 days after the operation. One month later, patients were permitted to perform partial-weightbearing walking, and conventional radiographs were obtained, including lateral and AP patellar radiographs. Eight weeks after treatment, patients were permitted to perform full ambulation. Patients were reexamined at 1, 2, 3, 6, and 12 months after the operation to observe the fracture healing. Fracture healing was defined as when a patient had no local pain or tenderness, the ability to walk well without help, and evidence of trabecular bone growing across the fracture line [15].

One year after surgery, the knee function was evaluated according to the Iowa knee score criteria [11], which evaluates knee function in daily life, inquires about joint pain when weightbearing, walking gait, the existence of deformity or instability, and joint ROM. The total score is 100 points: 90 to 100 points is excellent, 80 to 89 points is good, 70 to 79 points is fair, and less than 70 points is poor.

Results were analyzed by SPSS $^{\circledR}$ statistical software (SPSS Inc, Chicago, IL, USA). The differences in internal fixation fracture healing time, knee function, and incidence of complications between the two groups were analyzed using univariate analysis. Logistic regression was used to determine whether gender, age, injury mechanisms, and fracture classifications had an influence on differences between the two operation methods in terms of the postoperative interfragmentary gap, fracture healing time, complications, and knee function. The logistic regression determining which of the variables remained significant as they relate to the others was considered significantly different when $\mathrm{p}<0.05$.

\section{Results}

There was no difference $(\mathrm{p}<0.05)$ in the operation time between the $\mathrm{K}$-wire tension band group (mean, 48 minutes; range, 40-60 minutes) and cable-cannulated screw tension band group (mean, 55 minutes; range, 45-70 minutes).

Five patients in the K-wire tension band group experienced skin irritation generated by wire tails after the operation; eight patients had K-wire tension band loosening and migration, and in five of these, the fracture healed with the protection of a brace and the internal fixation was removed 1 year later. Three patients with internal fixation loosening and fracture displacement required a second operation, which was performed directly after the fracture displacement was discovered (Fig. 2). In the cable-cannulated screw tension band group, no complications were observed after the operations. Fractures healed well, and internal fixation showed no loosening or fractures.

The mean postoperative fracture spacing of the articular surface was larger $(\mathrm{p}=0.001)$ in the $\mathrm{K}$-wire tension band 

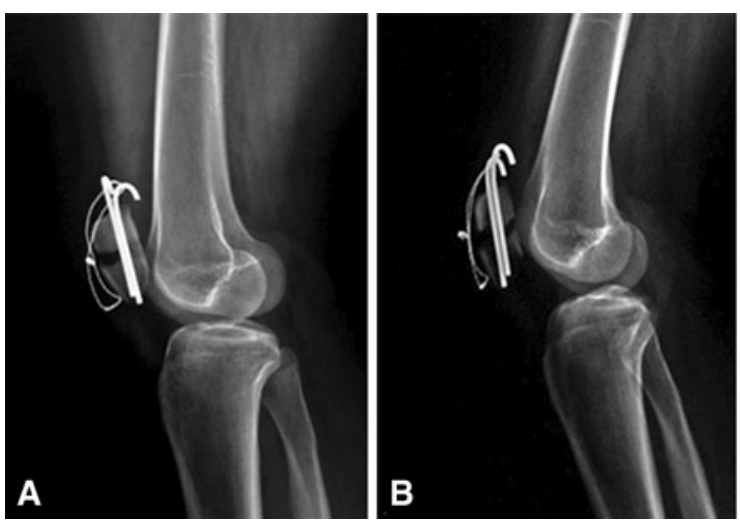

Fig. 2A-D A 34-year-old woman with a patellar fracture had K-wire tension band fixation failure. (A) A lateral radiograph shows the transverse patellar fracture 1 month after K-wire tension band fixation with tension band loosening. (B) A lateral radiograph taken 2 months postoperatively shows the fracture has not healed. (C) A lateral

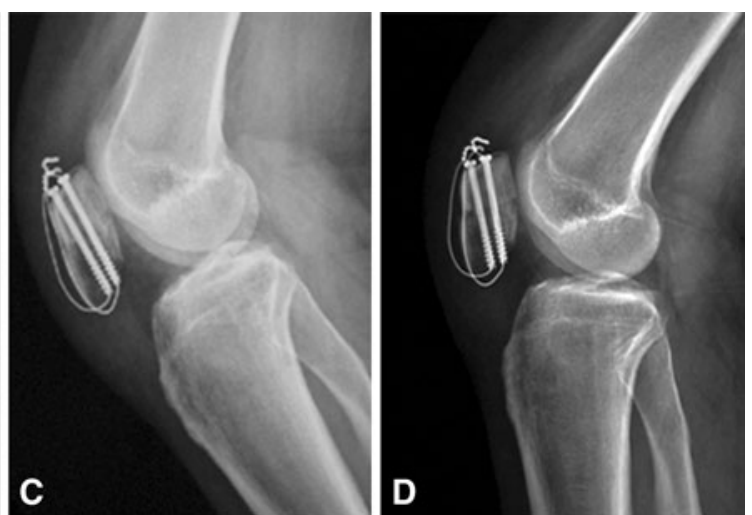

radiograph shows the patella fracture after revision with a titanium cable-cannulated screw tension band. (D) A lateral radiograph taken 2 months after the second operation shows fracture healing and recovery of knee function.

Table 2. Results of the two groups

\begin{tabular}{|c|c|c|c|c|}
\hline Variables & $\begin{array}{l}\text { Modified Kirschner } \\
\text { wire tension band }\end{array}$ & $\begin{array}{l}\text { Titanium cable-cannulated } \\
\text { screw tension band }\end{array}$ & Chi square/t value & p Value \\
\hline \multicolumn{5}{|l|}{ Postoperative interfragmentary gap } \\
\hline $0 \mathrm{~mm}$ & 33 & 47 & 16.14 & $<0.001$ \\
\hline$\geq 1 \mathrm{~mm}$ & 19 & 2 & & \\
\hline Knee function* & & & & $0.01^{*}$ \\
\hline $90-100$ & 36 & 45 & & \\
\hline $80-89$ & 9 & 4 & & \\
\hline $70-79$ & 4 & 0 & & \\
\hline$\leq 69$ & 3 & 0 & & \\
\hline Fracture healing time (months) ${ }^{\dagger}$ & $2.33 \pm 1.15$ & $2.00 \pm 0$ & 2.050 & 0.046 \\
\hline \multicolumn{5}{|l|}{ Complications } \\
\hline 0 & 41 & 49 & & \\
\hline 1 & 10 & 0 & & $<0.001^{*}$ \\
\hline 2 & 1 & 0 & & \\
\hline
\end{tabular}

* Iowa knee score [11] (total score $=100$ points): $90-100=$ excellent; $80-89=$ good; $70-79=$ fair; $\leq 69=$ poor; ${ }^{\dagger}$ values are expressed as mean $\pm \mathrm{SD}$; the remaining values are expressed as number of patients; "Fisher's exact test.

group $(0.63 \mathrm{~mm}$; range, $0-3 \mathrm{~mm})$ than in the cablecannulated screw tension band group $(0.04 \mathrm{~mm}$; range, 0-2 mm) (Table 2). The postoperative interfragmentary gaps in the tension band group were $2 \mathrm{~mm}$ in 10 patients and $3 \mathrm{~mm}$ in two patients (Fig. 3), whereas no patient's interfragmentary gap was greater than $2 \mathrm{~mm}$ in the titanium cable-cannulated screw tension band group.

The mean fracture healing time was longer $(\mathrm{p}=0.046)$ in the $\mathrm{K}$-wire tension band group (2.33 months; range, 2-4 months) than in the cable-cannulated screw tension band group (2.00 months) (Table 2) (Fig. 4).

According to the Iowa score criteria [11], the knee function scores were better $(p=0.01)$ in the titanium cable-cannulated screw tension band group (excellent,
45 patients; good, four; fair and poor, none) than in the modified K-wire tension band group (excellent, 36 patients; good, nine; fair, four; poor, three) (Table 2).

Logistic regression showed postoperative interfragmentary gap and knee function were associated with the operation method $(\mathrm{p}=0.0001$ and $\mathrm{p}=0.006$, respectively), with the cable-cannulated screw tension band group being superior. Slower healing time was associated with $(\mathrm{p}=0.013)$ AO/OTA $\mathrm{C} 2$ fractures but not with $(\mathrm{p}=0.997)$ the operation method. Because there was no main complication in the cable-cannulated screw tension band group, the logistic regression analysis could not show a significant association with the operation method (odds ratio $=0.000)($ Table 3$)$. 
Fig. 3A-C A 45-year-old woman with a patellar fracture was treated with $\mathrm{K}$-wire tension band fixation. (A) Her preoperative lateral radiograph shows a transverse patellar fracture. (B) A lateral radiograph taken after $\mathrm{K}$-wire tension band fixation shows the fracture line with levels and gaps. (C) A lateral radiograph show the status 2 months postoperatively.
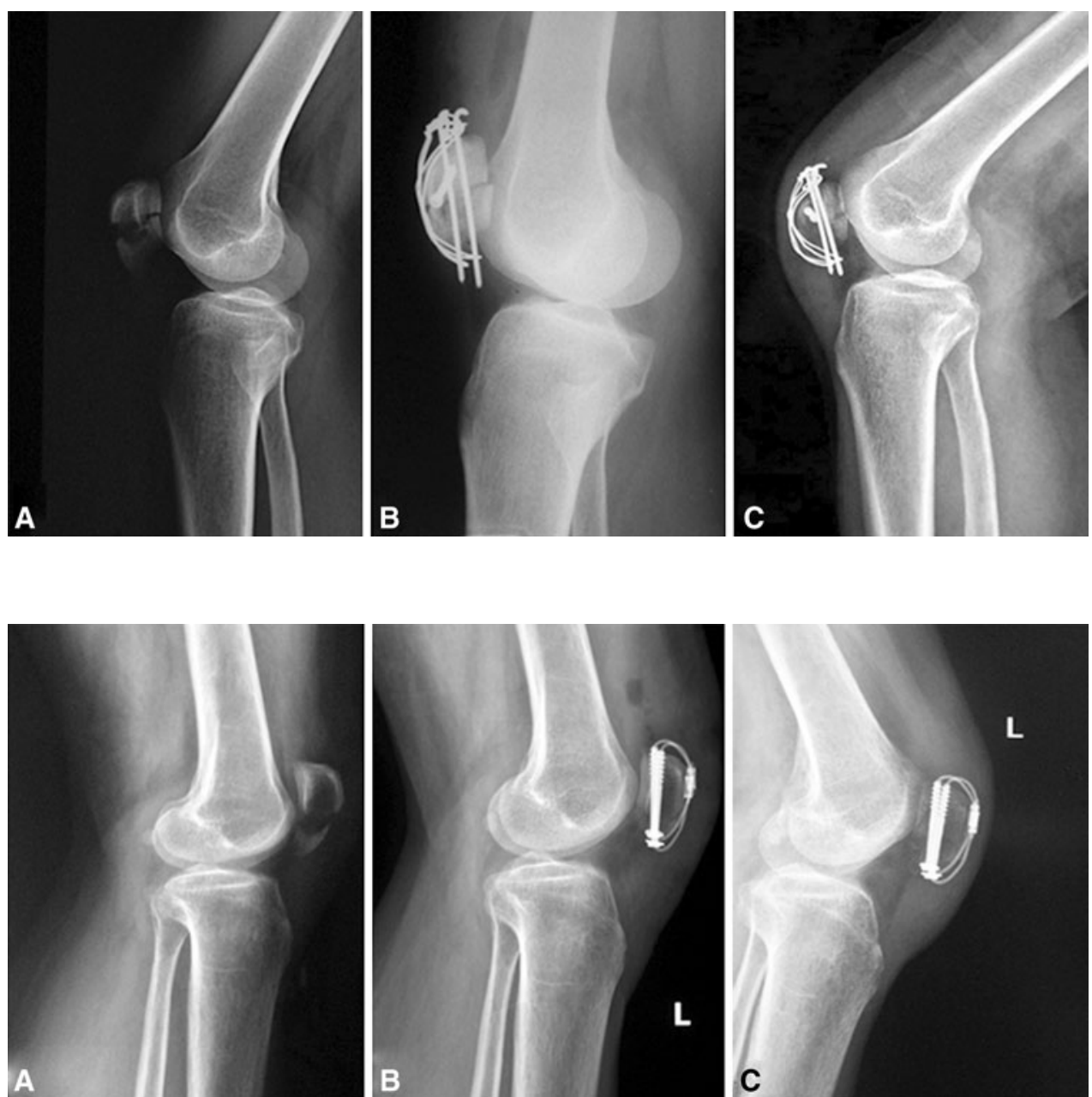

Fig. 4A-C A 35-year-old man with a patellar fracture underwent fracture fixation with a titanium cable-cannulated screw tension band. (A) His preoperative lateral radiograph shows a transverse patellar fracture. (B) A lateral radiograph shows the patella fracture after titanium cable-cannulated screw tension band fixation. (C) A lateral radiograph taken 2 months postoperatively shows fracture healing.

Table 3. Results of logistic regression analysis*

\begin{tabular}{|c|c|c|c|c|c|c|c|c|}
\hline \multirow[t]{2}{*}{ Variable } & \multicolumn{2}{|c|}{ Postoperative interfragmentary gap } & \multicolumn{2}{|c|}{ Complications } & \multicolumn{2}{|c|}{ Healing time } & \multicolumn{2}{|c|}{ Knee function } \\
\hline & Odds ratio & $\mathrm{p}$ Value & Odds ratio & $\mathrm{p}$ Value & Odds ratio & $\mathrm{p}$ Value & Odds ratio & $\mathrm{p}$ Value \\
\hline Gender (male) & 1.187 & 0.716 & 1.181 & 0.818 & 1.683 & 0.507 & 0.896 & 0.806 \\
\hline Age $(>50$ years $)$ & 0.398 & 0.141 & 0.970 & 0.159 & 1.005 & 0.849 & 0.984 & 0.263 \\
\hline Injury (slip and fall) & $5.777 \mathrm{E} 8$ & 0.999 & 5.618 & 0.132 & 0.000 & 0.999 & 3.568 & 0.168 \\
\hline AO/OTA classification (34-C2) & 0.557 & 0.342 & 0.821 & 0.827 & 6.997 & 0.013 & 2.253 & 0.093 \\
\hline $\begin{array}{l}\text { Operation method (modified } \\
\text { Kirschner wire tension band) }\end{array}$ & 17.845 & 0.0001 & 0.000 & 0.997 & 0.000 & 0.997 & 3.380 & 0.006 \\
\hline
\end{tabular}

* Variables included: gender (male, female); age ( $\leq 50$ years, $>50$ years), injury (slip and fall, car accident), AO/OTA classification (34-C1, 34-C2), operation method (titanium cable-cannulated screw tension band, modified K-wire tension band), postoperative interfragmentary gap $(0 \mathrm{~mm}, \geq 1 \mathrm{~mm})$, complications (loosening of implant, failure of fixation, skin irritation), healing time ( $\leq 2 \mathrm{months},>2 \mathrm{months})$, knee function (excellent, less than excellent).

\section{Discussion}

Selecting the appropriate and effective method and materials for treating patellar fractures is a tough challenge for orthopaedists $[1,6-8,12,13,15]$. Although the modified tension band technique (eg, tension band supplemented by longitudinal K-wires) has long been the mainstay for fixation of transverse fractures of the patella $[1,4-6,8,13$, $14]$, it is not without shortcomings. We therefore compared an alternative operative method, the titanium cable-cannulated screw tension band technique, with the modified tension band technique to see whether this 
technique could (1) achieve better reduction and direct interfragmentary compression force; (2) shorten fracture healing time; (3) decrease complications, such as loosening of implants and skin irritations; and (4) achieve better knee function.

There are several limitations of this study. First, the decision to treat patients with either a modified tension band or titanium cable-cannulated screw tension band was subject to selection bias as this was not a randomized study. Second, the degree to which the titanium cable or the steel wire was tightened was not standardized. As such, we could not be sure that the tension was equal in any of the patients, which might have had an influence on the interfragmentary pressure and tension band force. Finally, 12 patients were lost to followup, which might have influenced the final result.

The cable-cannulated screw tension band was better than the modified $\mathrm{K}$-wire tension band in fracture reduction. In the modified tension band group, the postoperative interfragmentary gaps were $2 \mathrm{~mm}$ in 10 patients and $3 \mathrm{~mm}$ in two patients after surgery, whereas no patient's interfragmentary gap was greater than $2 \mathrm{~mm}$ in the titanium cable-cannulated screw tension band group. As the support of a tension band, the K-wire itself cannot generate reduction and compression effects on fractured bones [1]. Rather, the reduction and compression effects on the patellar fracture line are generated by tightening the wires from the superior pole to the lower pole $[1,4,15]$. However, the flexibility of K-wires will counteract a part of the force that should act on the fractured bone. When using a cable-cannulated screw tension band the primary pressure is achieved by the two cannulated lag screws [2] and the titanium cable, which is fixed through the cannulated screw and tightened and plays its role as a tension band. Because the acting force generated by the cannulated screws is perpendicular to the fracture $[2,9,14]$, the acting forces on the patellar articular and anterior surfaces are consistent, which is different from a modified $\mathrm{K}$-wire tension band (Fig. 5).

Loosening of the implant is considered the main complication of patellar fracture fixation. In our study, eight patients in the modified K-wire band group had loosening

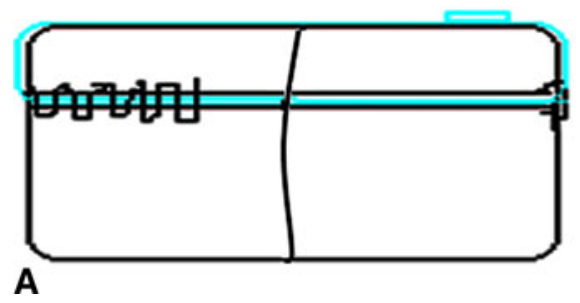

Fig. 5A-B The schematic diagrams illustrate titanium cable-cannulated screw tension band fixation of a patellar fracture. (A) The cannulated screw is located near the leading edge of the patella. of the K-wire tension band; three of them had separation of the fracture and required a second operation. Loss of fixation has been reported to occur in $0 \%$ to $20 \%$ of operatively treated patellar fractures $[6,9,11]$. The surface of a $\mathrm{K}$-wire is smooth, so loosening of $\mathrm{K}$-wire is inevitable with time. A previous study [1] suggested the reason for poor outcomes of a K-wire tension band was initial separation of the fracture fragments caused by properties of the wire and further separation resulting from the change in wire plasticity. Secondarily, the method of bending the $\mathrm{K}$-wire may influence the result. In our study, we bent only the proximal end of the K-wire. Some authors argue that bending of the distal wire ends decreases the risk of proximal wire migration with loss of reduction $[8,11,13$, 15]. Additionally, the technique used in twisting the wire may influence the stability of fixation. Some authors selected to twist the wire at two sites, rather than one $[7,11]$. We twisted the wire at only one site, which might have been a cause of implant failure. Some researchers use modified steel wires to increase the stability of a K-wire tension band; however, the effect still will be reduced by the flexibility of $\mathrm{K}$-wires $[8,12,13]$. The probability of titanium cable-cannulated screw loosening is very low owing to the dense cancellous bone of the patella and the fact that the distal end of the cannulated screw is threaded. In addition, the strong titanium cable tightly attaches to the patellar surface to limit the separation of fracture fragments as a tension band. We chose two individual tension band cables rather than one figure-of-eight band, because this allows tightening of the tension band independently.

Skin irritation is the main complication of the modified $\mathrm{K}$-wire tension band. Five patients experienced skin irritation in the modified K-wire tension band group, whereas no patients in the cable-cannulated screw tension band group had skin irritation. In some studies, symptoms attributable to wire irritation necessitated removal in approximately $15 \%$ of the cases [6,11]. $\mathrm{K}$ wire prominence and migration are the primary causes of skin irritation associated with this technique [7, 13]. With the cablecannulated screw tension band technique, the tail of the cannulated screw tightly attaches at the superior or inferior pole of the patella, and the screw threads are not exposed to

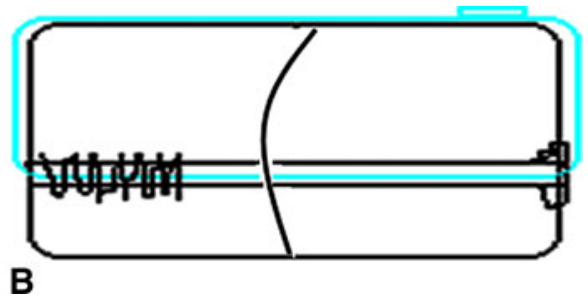

(B) The cannulated screw is located away from the leading edge of the patella. Regardless of the position of the cannulated screw, the titanium cable is close to the surface of the patella. 
the patellar surface; also, the diameter of the titanium cable is only $1.3 \mathrm{~mm}$, and it closely attaches to the patellar surface after being tightened reducing the risk of skin irritation and postoperative activity discomfort.

The Iowa knee score was better in the cable-cannulated screw tension band group. According to the logistic regression analysis, the healing time was associated with fracture classification, suggesting complicated fractures needed longer healing time, independent of the operative method. With respect to removal of the internal fixation, generally $\mathrm{K}$-wires should be removed 1 year after surgery except when patients refuse, whereas the cables and cannulated screws, both being titanium, rarely produce adverse effects such as skin irritation. However, removal of the titanium cannulated screws, if needed, is more difficult than a K-wire.

We developed an improved technique to remedy the shortcomings of the modified K-wire tension band in treating patellar fractures. A titanium cable-cannulated screw tension band can achieve good fracture reduction and healing with a low rate of complications. We therefore recommend its use in treating transverse patellar fractures.

Acknowledgments We thank Zeng Lin and Bao Yanping for assistance in the statistical analysis of our data and preparation of figures and tables for this article. We also acknowledge the considerable support from Niu Xiaoyan in the Department of Orthopaedics, Third Hospital of Peking University.

Open Access This article is distributed under the terms of the Creative Commons Attribution Noncommercial License which permits any noncommercial use, distribution, and reproduction in any medium, provided the original author(s) and source are credited.

\section{References}

1. Benjamin J, Bried J, Dohm M, McMurtry M. Biomechanical evaluation of various forms of fixation of transverse patellar fractures. J Orthop Trauma. 1987;1:219-222.
2. Berg EE. Open reduction internal fixation of displaced transverse patella fractures with figure-eight wiring through parallel cannulated compression screws. J Orthop Trauma. 1997;11:573-576.

3. Carpenter JE, Kasman R, Matthews LS. Fractures of the patella. Instr Course Lect. 1994;43:97-108.

4. Carpenter JE, Kasman RA, Patel N, Lee ML, Goldstein SA. Biomechanical evaluation of current patella fracture fixation techniques. J Orthop Trauma. 1997;11:351-356.

5. Cramer KE, Moed BR. Patellar fractures: contemporary approach to treatment. J Am Acad Orthop Surg. 1997;5:323-331.

6. Fortis AP, Milis Z, Kostopoulos V, Tsantzalis S, Kormas P, Tzinieris N, Boudouris T. Experimental investigation of the tension band in fractures of the patella. Injury. 2002;33:489493.

7. Gosal HS, Singh P, Field RE. Clinical experience of patellar fracture fixation using metal wire or non-absorbable polyester: a study of 37 cases. Injury. 2001;32:129-135.

8. John J, Wagner WW, Kuiper JH. Tension-band wiring of transverse fractures of patella: the effect of site of wire twists and orientation of stainless steel wire loop: a biomechanical investigation. Int Orthop. 2007;31:703-707.

9. Luna-Pizarro D, Amato D, Arellano F, Hernandez A, Lopez-Rojas P. Comparison of a technique using a new percutaneous osteosynthesis device with conventional open surgery for displaced patella fractures in a randomized controlled trial. J Orthop Trauma. 2006;20:529-535.

10. Marsh JL, Slongo TF, Agel J, Broderick JS, Creevey W, DeCoster TA, Prokuski L, Sirkin MS, Ziran B, Henley B, Audigé L. Fracture and dislocation classification compendium - 2007: Orthopaedic Trauma Association classification, database and outcomes committee. J Orthop Trauma. 2007;21(10 suppl): S1-S133.

11. Merchant TC, Dietz FR. Long-term follow-up after fractures of the tibial and fibular shafts. J Bone Joint Surg Am. 1989;71: 599-606.

12. Schnabel B, Scharf M, Schwieger K, Windolf M, Pol B, Braunstein V, Appelt A. Biomechanical comparison of a new staple technique with tension band wiring for transverse patella fractures. Clin Biomech (Bristol, Avon). 2009;24:855-859.

13. Smith ST, Cramer KE, Karges DE, Watson JT, Moed BR. Early complications in the operative treatment of patella fractures. J Orthop Trauma. 1997;11:183-187.

14. Tandogan RN, Demirors H, Tuncay CI, Cesur N, Hersekli M. Arthroscopic-assisted percutaneous screw fixation of select patellar fractures. Arthroscopy. 2002;18:156-162.

15. Wu CC, Tai CL, Chen WJ. Patellar tension band wiring: a revised technique. Arch Orthop Trauma Surg. 2001;121:12-16. 